\title{
EXAMPLES OF REDUCIBLE AND FINITE DEHN FILLINGS
}

\author{
SUNGMO KANG
}

\begin{abstract}
If a hyperbolic 3-manifold $M$ admits a reducible and a finite Dehn filling, the distance between the filling slopes is known to be 1. This has been proved recently by Boyer, Gordon and Zhang. The first example of a manifold with two such fillings was given by Boyer and Zhang. In this paper, we give examples of hyperbolic manifolds admitting a reducible Dehn filling and a finite Dehn filling of every type: cyclic, dihedral, tetrahedral, octahedral and icosahedral.
\end{abstract}

\section{INTRODUCTION}

Let $M$ be a compact connected orientable 3-manifold with a torus boundary component $\partial_{0} M$ and $r$ a slope, the isotopy class of an essential simple closed curve, on $\partial_{0} M$. The manifold obtained by $r$-Dehn filling on $M$ is defined to be $M(r)=M \cup V$, where $V$ is a solid torus glued to $M$ along $\partial_{0} M$ so that $r$ bounds a disk in $V$.

We say that $M$ is hyperbolic if $M$ with its torus boundary components removed has a complete hyperbolic structure of finite volume. For a pair of slopes $r_{1}$ and $r_{2}$ on $\partial_{0} M$, the distance $\Delta\left(r_{1}, r_{2}\right)$ denotes their minimal geometric intersection number. For a hyperbolic manifold $M$, if both $M\left(r_{1}\right)$ and $M\left(r_{2}\right)$ fail to be hyperbolic, then the upper bounds for $\Delta\left(r_{1}, r_{2}\right)$ have been established in various cases. See [10]

We are interested in the case of reducible and finite Dehn fillings (i.e. a filling whose fundamental group is finite). The first step towards determining the least upper bound for $\Delta\left(r_{1}, r_{2}\right)$ in that case was achieved in [1, where the bound of 2 was established. Furthermore, in [1] it was shown that if $\Delta\left(r_{1}, r_{2}\right)=2$ then $H_{1}(M) \cong \mathbb{Z} \oplus \mathbb{Z}_{2}$ and the reducible Dehn filling is homeomorphic to $L(2,1) \# L(3,1)$. Recently, this special case was eliminated by Boyer, Gordon and Zhang [2], showing that $\Delta\left(r_{1}, r_{2}\right)=1$

There are five types of finite group that can occur as the fundamental group of a 3-manifold: cyclic, dihedral, tetrahedral, octahedral and icosahedral. See [3, Section 1] for the definition of these. It is known that all these types of finite group can be realized as the fundamental group of either a lens space or a Seifert fibered space over $S^{2}$ with three exceptional fibers of orders $a, b, c$ satisfying $1 / a+1 / b+1 / c>1$. The latter is denoted by $S^{2}(a, b, c)$. More precisely cyclic, dihedral, tetrahedral, octahedral and icosahedral types

Key words and phrases. Dehn filling; Reducible; Finite Dehn filling; Tangle filling. 
are the fundamental groups of a lens space, $S^{2}(2,2, n), S^{2}(2,3,3), S^{2}(2,3,4)$ and $S^{2}(2,3,5)$ respectively.

The following are the known examples of hyperbolic manifolds admitting a reducible and a finite Dehn filling so far;

(1) In 4, Example 7.8], Boyer and Zhang gave the first example of a manifold realizing the upper bound 1 . This manifold admits a reducible Dehn filling, a Dehn filling of cyclic type and a Dehn filling of dihedral type. See Sections 2, 3 for details.

(2) In [7, Section 4], Eudave-Muñoz and $\mathrm{Wu}$ gave an infinite family of hyperbolic manifolds admitting a reducible Dehn filling and a Dehn filling of cyclic type. See Section 2 for details.

(3) In [14, Section 4], Lee constructed a family of hyperbolic manifolds and showed that these manifolds admit an $S^{1} \times S^{2}$ Dehn filling and a toroidal Dehn filling with distance 2. However we observe that some manifolds in this family also admit a Dehn filling of icosahedral type. See Section 6 for details.

In this paper we give examples of all types of finite group. The technique of constructing examples is to use tangles and double branched covers. The double branched cover of a tangle is a 3-manifold whose boundary consists of tori. Also performing a rational tangle filling on a given tangle corresponds to performing a Dehn filling to the corresponding double branched cover since the double branched cover of a rational tangle is a solid torus. By using tangle arguments, we establish the following theorem.

Theorem 1.1. There are hyperbolic manifolds admitting a reducible Dehn filling and a finite Dehn filling of every type at distance 1: cyclic, dihedral, tetrahedral, octahedral and icosahedral.

This paper is organized as follows. In Section 2 we construct another infinite family of hyperbolic manifolds admitting a reducible Dehn filling (i.e. the connected sum of two lens spaces of arbitrary orders) and a Dehn filling of cyclic type (i.e. a lens space). In Section 3 we present hyperbolic manifolds which admit a reducible Dehn filling (i.e. the connected sum of two lens spaces) and a Dehn filling of dihedral type (i.e. $\left.S^{2}(2,2, n)\right)$. In Section 4 we give the first example of a hyperbolic manifold admitting a reducible Dehn filling (i.e. the connected sum of two lens spaces) and a Dehn filling of tetrahedral type (i.e. $S^{2}(2,3,3)$ ). In Section 5 we describe the first examples (infinitely many) of hyperbolic manifolds which admit a reducible Dehn filling (i.e. the connected sum of a lens space and a small Seifert fibered space) and a Dehn filling of octahedral type (i.e. $S^{2}(2,3,4)$ ). In Section 6 we give another example of a hyperbolic manifold admitting a reducible Dehn filling (i.e. the connected sum of two lens spaces) and a Dehn filling of icosahedral type (i.e. $\left.S^{2}(2,3,5)\right)$.

Throughout the paper, $S\left(a_{1}, a_{2}, \ldots, a_{n}\right)$ denotes a Seifert fibered space over a surface $S$ with $n$ exceptional fibers of orders $a_{1}, a_{2}, \ldots, a_{n}$, and $C(s, t)$ denotes the cable space as defined in [11, Section 3]. 


\section{CyClic Dehn fillings}

In this section, we show that there are hyperbolic manifolds which admit a reducible Dehn filling and a finite cyclic Dehn filling at distance 1.

The first example was given by Boyer and Zhang in [4, Example 7.8]. In their example $M=W(6)$, which is obtained by Dehn filling on one boundary component of the Whitehead link with slope 6 , admits a reducible Dehn filling $M(1)(=L(3,1) \# L(2,1))$ and a cyclic Dehn filling $M(1 / 0)(=L(6,1))$.

The first infinite family of hyperbolic manifolds admitting a cyclic Dehn filling and a reducible Dehn filling was given by Eudave-Muñoz and $\mathrm{Wu}$ in [7. Section 4]. In their examples, the hyperbolic manifolds $M_{p}, p \geq 2$ allow a 0 -Dehn filling $M_{p}(0)$, which is the lens space $L((p-1)(p+3)+1, p+3)$, and a $1 / 3$-Dehn filling $M_{p}(1 / 3)$, which is the reducible manifold $L(3,1) \# L(2,1)$. We can observe that Boyer and Zhang's manifold $W(6)$ belongs to this family $M_{p}$ i.e. $W(6)=M_{2}$. Note that the reducible Dehn filling is always $L(3,1) \# L(2,1)$ regardless of $p$. So it is natural to try to find some examples realizing the connected sum of two lens spaces of arbitrary orders.

We will construct hyperbolic manifolds which admit a Dehn filling that is a connected sum of lens spaces of arbitrary orders and a lens space Dehn filling. Consider the tangles $\mathcal{T}_{p, q}$ illustrated in Figure 1 with $p \geq 2$ and $q \geq 4$. Let $\mathcal{T}_{p, q}(r)$ be the link obtained by filling an $r$-rational tangle to $\mathcal{T}_{p, q}$, $M_{p, q}$ the double branched cover of the tangle $\mathcal{T}_{p, q}$ and $M_{p, q}(r)$ the double branched cover of $S^{3}$ branched along $\mathcal{T}_{p, q}(r)$.

Lemma 2.1. The manifolds $M_{p, q}$ admit the following Dehn fillings.

(1) $M_{p, q}(0)=L(p, 1) \# L(q-2,1)$;

(2) $M_{p, q}(\infty)$ is the lens space $L((3 p+2)(-2 q+1)+6,(3 p+2) q-3)$;

(3) $M_{p, q}(-1)$ is an irreducible, toroidal and non-Seifert fibered manifold.

Proof. The tangles $\mathcal{T}_{p, q}(0), \mathcal{T}_{p, q}(\infty), \mathcal{T}_{p, q}(-1)$ are shown in Figure 1, The result now follows by taking the double branched cover of $S^{3}$ branched along the corresponding links.

Theorem 2.2. The manifolds $M_{p, q}$ are hyperbolic manifolds admitting two Dehn fillings $M_{p, q}\left(r_{1}\right)$ and $M_{p, q}\left(r_{2}\right)$ such that $M_{p, q}\left(r_{1}\right)$ is the connected sum of two lens spaces of arbitrary orders, $M_{p, q}\left(r_{2}\right)$ is a lens space and $\Delta\left(r_{1}, r_{2}\right)=1$.

Proof. Let $r_{1}=0$ and $r_{2}=\infty$. Then $\Delta\left(r_{1}, r_{2}\right)=1$ and by Lemma 2.1. $M\left(r_{1}\right)$ is the connected sum of two lens spaces of arbitrary orders and $M\left(r_{2}\right)$ is a lens space. To complete the proof, we need to show that $M_{p, q}$ is hyperbolic i.e. $M_{p, q}$ is irreducible, $\partial$-irreducible, non-Seifert fibered and atoroidal. However this follows from Lemmas 2.3-2.6 below.

Lemma 2.3. $M_{p, q}$ is irreducible, $\partial$-irreducible and not Seifert fibered.

Proof. First, we show that $M_{p, q}$ is irreducible. Suppose $M_{p, q}$ is reducible. Then it contains a separating essential sphere since $M_{p, q}(\infty)$ is a lens space. 

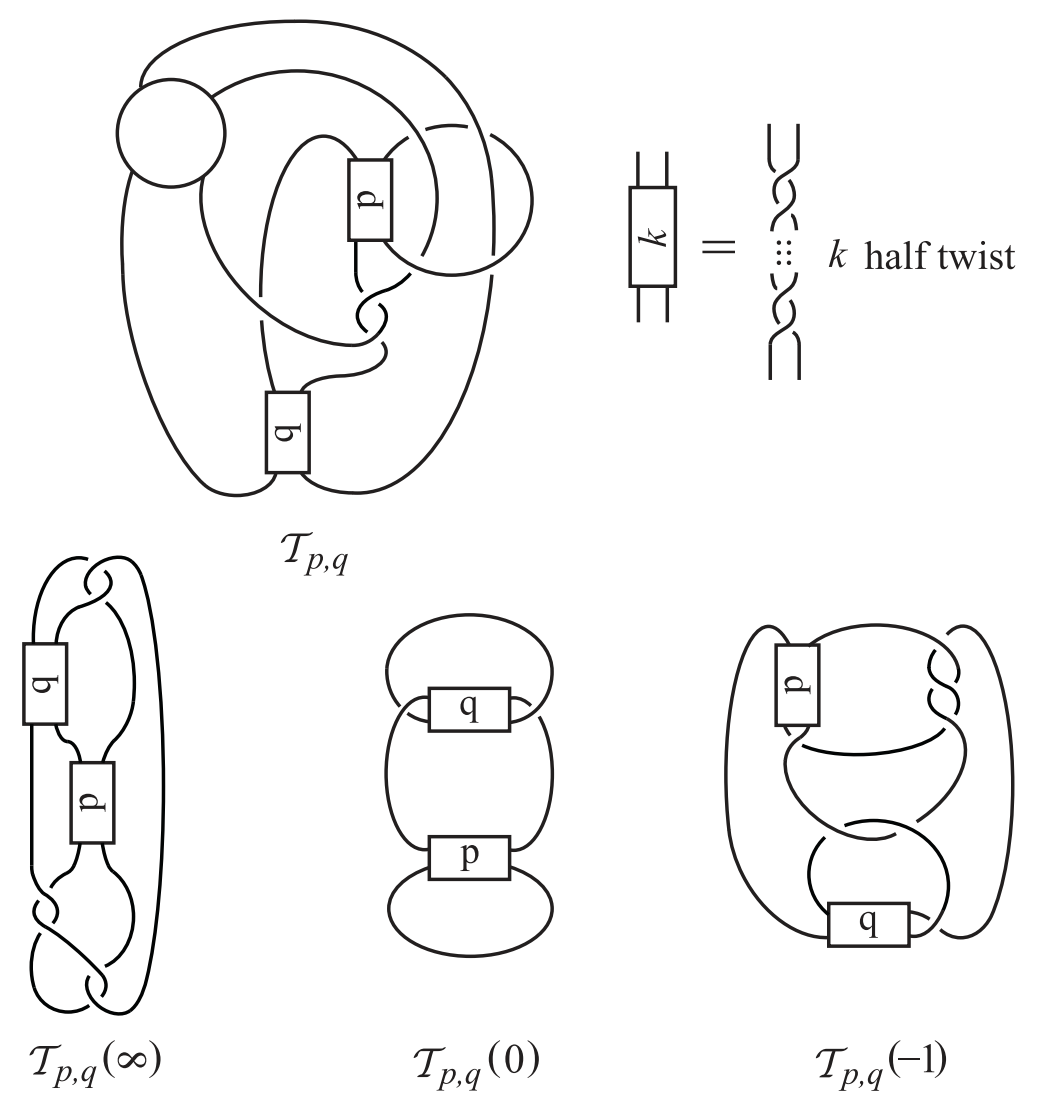

Figure 1. Cyclic Dehn fillings.

Therefore $M_{p, q}=X \# Y$ where $Y$ contains the torus boundary of $M_{p, q}$ and $X \neq S^{3}$. Note that if there are two slopes $r$ and $r^{\prime}$ such that $M_{p, q}(r)$ and $M_{p, q}\left(r^{\prime}\right)$ are prime, then $M_{p, q}(r)=M_{p, q}\left(r^{\prime}\right)(=X)$ since $Y(r)$ and $Y\left(r^{\prime}\right)$ must be $S^{3}$. However, by Lemma $2.1 M_{p, q}(\infty)$ and $M_{p, q}(-1)$ are both prime and distinct, which implies that $M_{p, q}$ is irreducible.

Suppose $M_{p, q}$ is a Seifert fibered space. Then $M_{p, q}(r)$ is Seifert fibered for all but at most one $r$, for which $M_{p, q}(r)$ is reducible. However $M_{p, q}(-1)$ is irreducible and not Seifert fibered, which is a contradiction. Therefore $M_{p, q}$ is not a Seifert fibered space.

Suppose $M_{p, q}$ is $\partial$-reducible. After $\partial$-compression, the torus boundary becomes a sphere which must bound a 3 -ball since $M_{p, q}$ is irreducible. This implies that $M_{p, q}$ is a solid torus, which is a Seifert fibered space, a contradiction.

To prove that $M_{p, q}$ is atoroidal, we need the following lemmas.

Lemma 2.4. Let $M\left(\neq T^{2} \times I\right)$ be an irreducible and $\partial$-irreducible 3-manifold with a torus boundary component $T_{0}$, and let $\alpha, \beta$ be slopes on $T_{0}$ with $\Delta(\alpha, \beta) \geq 2$. Let $T_{1}$ be a torus in $\partial M-T_{0}$ which is incompressible in 
M. If $T_{1}$ is compressible in $M(\alpha)$ and $M(\beta)$, then $M$ is a cable space with cabling slope $\gamma$ satisfying $\Delta(\alpha, \gamma)=\Delta(\beta, \gamma)=1$.

Proof. This follows immediately from [6, Theorems 2.4.3 and 2.4.4].

Lemma 2.5. Let $M\left(\neq T^{2} \times I\right)$ be an irreducible and $\partial$-irreducible 3-manifold with a torus boundary component, and let $\alpha, \beta$ be slopes on $T_{0}$ with $\Delta(\alpha, \beta)=$ 1. Let $T_{1}$ be a torus in $\partial M-T_{0}$ which is incompressible in $M$. If $T_{1}$ is compressible in $M(\alpha)$ and $M(\beta)$, then either

(1) $M$ is a cable space $C(s, t), t \geq 2$ with cabling slope $\alpha$, say, and thus $M(\alpha)=S^{1} \times D^{2} \# L(t, s)$ and $M(\beta)=S^{1} \times D^{2}$. Furthermore if $r_{\alpha}, r_{\beta}$ are the slopes on $T_{1}$ corresponding to the meridians of the solid tori of $M(\alpha), M(\beta)$ respectively, then $\Delta\left(r_{\alpha}, r_{\beta}\right) \geq 2$; or

(2) $M(\alpha)$ and $M(\beta)$ are $S^{1} \times D^{2}$ and if $r_{\alpha}, r_{\beta}$ are the slopes on $T_{1}$ corresponding to the meridians of $M(\alpha), M(\beta)$ respectively, then $\Delta\left(r_{\alpha}, r_{\beta}\right) \geq 4$.

Proof. It follows from [7, Lemma 3.4] that either $M(\alpha)$ or $M(\beta)$ must be irreducible. We assume without loss of generality that $M(\beta)$ is irreducible. Since $T_{1}$ is compressible in $M(\beta)$ i.e. $M(\beta)$ is $\partial$-reducible, $M(\beta)=S^{1} \times D^{2}$. Therefore $\partial M$ consists of $T_{0}$ and $T_{1}$. Let $K_{\beta}$ be the core of the Dehn filling solid torus. Then $M(\alpha)$ is obtained by Dehn surgery on $K_{\beta}$ in the solid torus $M(\beta)$.

(1) Assume that $M(\alpha)$ is reducible. It follows from [8, Theorem 1.1] and [16. Theorem 6.1] that $M$ is a cable space $C(s, t)$ with cabling slope $\alpha$. Hence $M(\alpha)=S^{1} \times D^{2} \# L(t, s)$. Let $r_{\alpha}, r_{\beta}$ be the slopes on $T_{1}$ corresponding to the meridians of the solid tori summands of $M(\alpha), M(\beta)$ respectively. Since $M$ is a cable space, we can apply [11, Lemma 3.1] to get $\Delta\left(r_{\alpha}, r_{\beta}\right)=|t| \Delta(\alpha, \beta)$. Since $M \neq T^{2} \times I,|t| \geq 2$. Therefore $\Delta\left(r_{\alpha}, r_{\beta}\right) \geq 2$.

(2) Assume that $M(\alpha)$ is irreducible. Since $T_{1}$ is compressible in $M(\alpha)$ i.e. $M(\alpha)$ is $\partial$-reducible, $M(\alpha)=S^{1} \times D^{2}$. Then it follows from [8, Theorem 1.1] that $M$ is the exterior of a braid in a solid torus. Let $r_{\alpha}, r_{\beta}$ be the slopes on $T_{1}$ corresponding to the meridians of the solid tori $M(\alpha), M(\beta)$ respectively. We can apply [9, Lemma 3.3] to get $\Delta\left(r_{\alpha}, r_{\beta}\right) \geq w^{2}$ where $w$ is the winding number of $K_{\beta}$ in the solid torus $M(\beta)$. Since $M \neq T^{2} \times I$, $|w| \geq 2$. We are done.

Lemma 2.6. $M_{p, q}$ is atoroidal.

Proof. Suppose on the contrary that $M_{p, q}$ is not atoroidal i.e. it contains an essential torus $F$. Note that $F$ is separating since $M_{p, q}(\infty)$ is a lens space. Let $M_{p, q}=A \cup_{F} B$ where $B$ contains $\partial M_{p, q}$. Since $M_{p, q}$ is irreducible and $\partial$-irreducible, $A$ and $B$ are also irreducible and $\partial$-irreducible. Lemma 2.1 implies that $M_{p, q}(0)$ and $M_{p, q}(\infty)$ are atoroidal. Hence $F$ must be compressible in both $B(0)$ and $B(\infty)$. Apply Lemma 2.5 to $B$. Then there are two cases to consider. 
Case 1: $B$ is a cable space $C(s, t)$. First assume that $\infty$ is the cabling slope. Then $B(\infty)=S^{1} \times D^{2} \# L(t, s)$ and $B(0)=S^{1} \times D^{2}$. Let $r_{\infty}, r_{0}$ be the slopes on $F$ corresponding to the meridians of the solid tori of $B(\infty), B(0)$ respectively. Then $\Delta\left(r_{\infty}, r_{0}\right) \geq 2$. Observe that $M(\infty) \cong A\left(r_{\infty}\right) \# L(t, s)$ and $M(0) \cong A\left(r_{0}\right)$. Since $M_{p, q}(\infty)$ is a lens space and $M_{p, q}(0)$ is the connected sum of two lens spaces, $A\left(r_{\infty}\right) \cong S^{3}$ and $A\left(r_{0}\right)$ is reducible. Then $\Delta\left(r_{\infty}, r_{0}\right) \leq 1$ by [12, a contradiction.

Assume that 0 is the cabling slope of $B=C(s, t)$. Then $B(0)=S^{1} \times$ $D^{2} \# L(t, s)$ and $B(\infty)=S^{1} \times D^{2}$ and $\Delta\left(r_{0}, r_{\infty}\right) \geq 2$. Moreover, $M(0) \cong$ $A\left(r_{0}\right) \# L(t, s)$ and $M(\infty) \cong A\left(r_{\infty}\right)$. By Lemma 2.1. $A\left(r_{0}\right)$ and $A\left(r_{\infty}\right)$ are lens spaces. By the Cyclic Surgery Theorem of [6], $A$ must be a Seifert fibered space. Consider the -1 -Dehn filling $M(-1)$, which is an irreducible, toroidal and non-Seifert fibered 3-manifold. Since $B=C(s, t)$ with the cabling slope 0 and $\Delta(0,-1)=1, B(-1)$ is a solid torus. Thus $M(-1) \cong$ $A\left(r_{-1}\right)$ where $r_{-1}$ is the slope on $F$ corresponding to the meridian of the solid torus $B(-1)$. Since $A$ is a Seifert fibered space, $A\left(r_{-1}\right)(\cong M(-1))$ is either a Seifert fibered space or a reducible manifold, a contradiction.

Case 2: $B(\infty)$ and $B(0)$ are $S^{1} \times D^{2}$. Then $M(\infty) \cong A\left(r_{\infty}\right)$ and $M(0) \cong$ $A\left(r_{0}\right)$. By Lemma 2.1, $A\left(r_{\infty}\right)$ is a lens space and $A\left(r_{0}\right)$ is reducible. Also by Lemma 2.5] $\Delta\left(r_{\infty}, r_{0}\right) \geq 4$. This is a contradiction to [4, Theorem 1.2].

\section{Dihedral Dehn fillings}

In this section, we show that there are hyperbolic manifolds which admit a reducible Dehn filling and a dihedral Dehn filling i.e. a Dehn filling of type $S^{2}(2,2, n)$ at distance 1 .

The first example was Boyer and Zhang's manifold $M=W(6)$ as described in Section 2. The manifold $M=W(6)$ admits a Dehn filling $M(2)$ which is $S^{2}(2,2,4)$, i.e. of dihedral type.

We present infinitely many examples of such hyperbolic manifolds. Consider the tangles $\mathcal{T}_{p, q}$ with $p, q \geq 3$ illustrated in Figure 2, Then 0-rational tangle filling on $\mathcal{T}_{p, q}, \mathcal{T}_{p, q}(0)$, is a connected sum of two 2-bridge links. Thus the double branched cover $M_{p, q}(0)$ is a connected sum of two lens spaces. $\infty$-rational tangle filling on $\mathcal{T}_{p, q}, \mathcal{T}_{p, q}(\infty)$, is a Montesinos link, whose double branched cover is a Seifert fibered space over $S^{2}$ with three exceptional fibers. More precisely, we get the following.

Lemma 3.1. The manifolds $M_{p, q}$ admit the following Dehn fillings.

(1) $M_{p, q}(0)=L(p, 1) \# L(2 q+1,1)$;

(2) $M_{p, q}(\infty)=S^{2}(2,2, n)$ where $n=2 p q-p-2$.

The main theorem of this section is the following.

Theorem 3.2. The manifolds $M_{p, q}$ are hyperbolic manifolds, admitting two Dehn fillings $M_{p, q}\left(r_{1}\right)$ and $M_{p, q}\left(r_{2}\right)$, such that $M_{p, q}\left(r_{1}\right)$ is the connected sum of two lens spaces, $M_{p, q}\left(r_{2}\right)$ is $S^{2}(2,2, n)$ and $\Delta\left(r_{1}, r_{2}\right)=1$. 

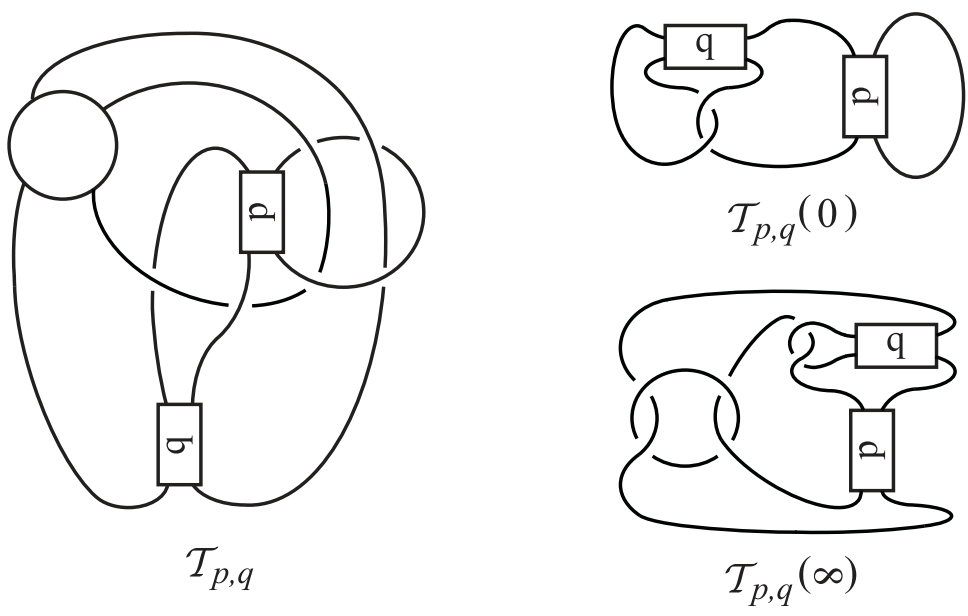

Figure 2. Dihedral Dehn fillings.

Proof. This follows immediately from Lemmas 3.1, 3.3 and 3.4 .

Lemma 3.3. $M_{p, q}$ is irreducible, $\partial$-irreducible and not Seifert fibered.

Proof. First, we show that $M_{p, q}$ is irreducible. Suppose $M_{p, q}$ is reducible i.e. it contains an essential sphere $S$. Then $S$ must be separating since $M_{p, q}(\infty)$ is irreducible. The sphere $S$ decomposes $M_{p, q}$ as $X \# Y$ where $Y$ contains $\partial M_{p, q}$. Then $M_{p, q}(\infty)=X \# Y(\infty)$. Since $M_{p, q}(\infty)\left(=S^{2}(2,2, n)\right)$ is irreducible, $Y(\infty)$ must be $S^{3}$. Hence $X \cong S^{2}(2,2, n)$.

On the other hand, 0 -Dehn filling $M_{p, q}(0)$ is $X \# Y(0)$. If $S$ is inessential in $M_{p, q}(0)$ i.e. $Y(0)$ is $S^{3}$, then $M_{p, q}(0) \cong X$, which is the connected sum of two lens spaces, a contradiction to $X=S^{2}(2,2, n)$. If $S$ is essential in $M_{p, q}(0)$, then by the uniqueness of the prime decomposition of a 3-manifold $X$ must be a lens space, a contradiction.

Secondly, we show that $M_{p, q}$ is not a Seifert fibered space. Suppose $M_{p, q}$ is a Seifert fibered space. Since $M_{p, q}(\infty)$ is $S^{2}(2,2, n), M_{p, q}$ is either $D^{2}(a, b)$ , $D^{2}(a, b, c)$ or $M^{2}(c)$ where one of $a, b$ is 2 (let $a=2$ ) and $M^{2}$ is a Möbius band. Observe that $M_{p, q}(r)$ is Seifert fibered for all but at most one $r$, for which $M_{p, q}(r)$ is reducible. When $M_{p, q}(r)$ is reducible, $r$ corresponds to the slope of the Seifert fiber of $M_{p, q}$. Lemma 3.1 shows that 0 is the slope of the Seifert fiber of $M_{p, q}$. Considering the fundamental group of $D^{2}(a, b)$, $D^{2}(a, b, c)$, or $M^{2}(c)$ and Dehn filling with slope of the Seifert fiber, it follows that $\pi_{1}\left(M_{p, q}(0)\right)$ is isomorphic to either $\mathbb{Z}_{a} * \mathbb{Z}_{b}, \mathbb{Z}_{a} * \mathbb{Z}_{b} * \mathbb{Z}_{c}$ or $\mathbb{Z} * \mathbb{Z}_{c}$ with $a=2$. This is a contradiction since $\pi_{1}\left(M_{p, q}(0)\right)=\mathbb{Z}_{p} * \mathbb{Z}_{2 q+1}$ where $p$ and $2 q+1$ are greater than 2 .

$\partial$-irreducibility of $M_{p, q}$ follows from irreducibility and the fact that $M_{p, q}$ is not Seifert fibered.

Lemma 3.4. $M_{p, q}$ is atoroidal. 

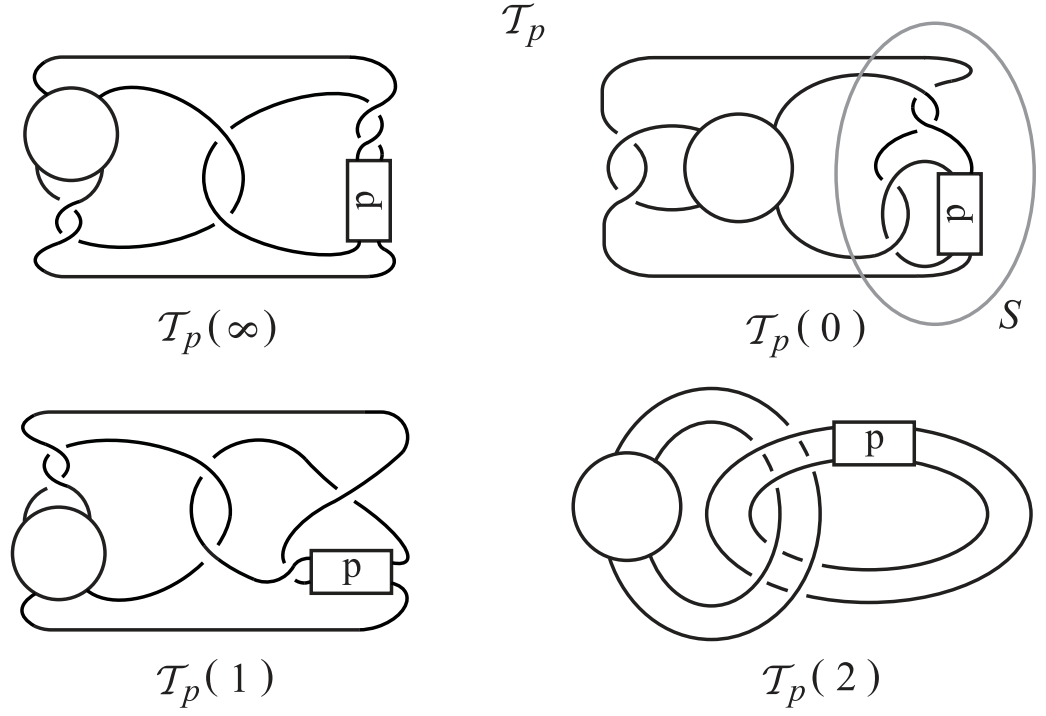

FiguRE 3. Rational tangle fillings on $\mathcal{T}_{p}$ obtained from $\mathcal{T}_{p, q}$ by removing $1 / q$-rational tangle.

Proof. Suppose on the contrary that $M_{p, q}$ is toroidal. We consider the tangle $\mathcal{T}_{p}$ with two tangle spheres $\mathcal{S}_{0}, \mathcal{S}_{1}$ depicted in Figure 3, Let $N_{p}, \partial_{0} N_{p}, \partial_{1} N_{p}$ be the double branched covers of $\mathcal{T}_{p}, \mathcal{S}_{0}, \mathcal{S}_{1}$ respectively. Then observe that the tangle $\mathcal{T}_{p, q}$ in Figure 2 is obtained from $\mathcal{T}_{p}$ by $1 / q$-rational tangle filling on $\mathcal{S}_{1}$. From the viewpoint of the double branched cover, $M_{p, q}$ is obtained from $N_{p}$ by $1 / q$-Dehn filling on $\partial_{1} N_{p}$ i.e. $M_{p, q}=N_{p}(1 / q)$. Hence $N_{p}(1 / q)$ is toroidal.

We perform rational tangle fillings on $\mathcal{T}_{p}$ along $\mathcal{S}_{1}$. Several rational tangle fillings are shown in Figure 3 . Then we get the corresponding double branched covers as follows; $N_{p}(\infty) \cong D^{2}(2, p+2), N_{p}(0) \cong C(1,2) \cup_{R}$ $D^{2}(2, p), N_{p}(1) \cong D^{2}(2, p-2)$ and $N_{p}(2) \cong Z \times S^{1}$ where $R$ is a torus corresponding to the Conway sphere $S$ as shown in Figure 3 and $Z$ is a once-punctured torus. Note that $N_{p}(0)$ is not Seifert fibered and $N_{p}(2)$ contains a non-separating essential torus. 
Claim. $N_{p}$ is hyperbolic.

Proof. Since $N_{p}(\infty)$ and $N_{p}(1)$ are distinct prime manifolds, $N_{p}$ is irreducible. Also, $N_{p}$ is not Seifert fibered because $N_{p}(1 / q)$ is an irreducible non-Seifert fibered space by Lemma 3.3. By irreducibility and the fact that it is not Seifert fibered, $N_{p}$ is $\partial$-irreducible. To complete the proof, we need only to show that $N_{p}$ is atoroidal.

Suppose $N_{p}$ contains an essential torus $F$. Then $F$ must be separating since $N_{p}(\infty)$ does not contain a non-separating torus or sphere. Let $N_{p}=$ $A \cup_{F} B$ with the filling torus $\partial_{1} N_{p} \subseteq B$.

Recall that $N_{p}(\infty) \cong D^{2}(2, p+2), N_{p}(1) \cong D^{2}(2, p-2), N_{p}(0) \cong C(1,2) \cup_{R}$ $D^{2}(2, p)$ and $N_{p}(2) \cong Z \times S^{1}$ contains a non-separating essential torus. Thus $F$ must be compressible in $B(\infty), B(1)$ and $B(2)$. Apply Lemma 2.5 to $B$ with slopes $\infty, 1,2$. Since $N_{p}(\infty), N_{p}(1)$ and $N(2)$ don't have a lens space summand, $\infty, 1$ and 2 can't be cabling slope, which implies that $B(\infty), B(1)$ and $B(2)$ are solid tori. Therefore $F$ separates the two boundaries $\partial_{0} N_{p}$, $\partial_{1} N_{p}$ of $N_{p}$. In other words, $A$ has the boundary $\partial_{0} N_{p}$ and $B$ has the boundary $\partial_{1} N_{p}$.

Suppose $F$ is incompressible in $N_{p}(0) \cong C(1,2) \cup_{R} D^{2}(2, p)$. Then $F$ is isotopic to $R$. Thus $A$ is homeomorphic to either $C(1,2)$ or $D^{2}(2, p)$. However $A$ has the boundary $\partial_{0} N_{p}$ and therefore $A \cong C(1,2)$. Since $B(2)$ is a solid torus, $N_{p}(2)$ can be obtained from $C(1,2)$ by Dehn filling on $F$. Hence $N_{p}(2)$ does not contain a non-separating torus, a contradiction. It follows that $F$ is compressible in $N_{p}(0)$ and thus in $B(0)$.

We have shown that $F$ is compressible in $B(\infty), B(0), B(1)$ and $B(2)$. Since $\Delta(0,2)=2$, it follows from Lemma 2.4 that $B$ is a cable space with cabling slope either $\infty$ or 1 . This implies that either $N_{p}(\infty)$ or $N_{p}(1)$ has a lens space summand, which is a contradiction. This completes the proof of the claim.

The claim says that $N_{p}$ is hyperbolic. However $N_{p}$ admits the two toroidal Dehn fillings $N_{p}(2), N_{p}(1 / q)$, and $\Delta(2,1 / q)=|2 q-1| \geq 5$ since $q \geq 3$. Since $N_{p}$ has two boundary components, [13, Theorem 1.1] implies that $N_{p}$ is homeomorphic to the exterior of the Whitehead sister link and in the two toroidal Dehn fillings every essential torus is separating. This is a contradiction because $N_{p}(2)$ contains a non-separating essential torus.

\section{Tetrahedral Dehn fillings}

In this section, we describe a hyperbolic manifold which admits a reducible Dehn filling and a tetrahedral Dehn filling, i.e. an $S^{2}(2,3,3)$-Dehn filling.

Let $\mathcal{T}$ be the tangle depicted in Figure 4. We perform 0,1 and $\infty$-rational tangle fillings on $\mathcal{T}$. See Figure 4. Then $\mathcal{T}(0)$ is the connected sum of two trefoil knots and $\mathcal{T}(1), \mathcal{T}(\infty)$ are Montesinos links. Let $M$ be the double branched cover of the tangle $\mathcal{T}$. Considering double branched covers, we have the following lemma. 

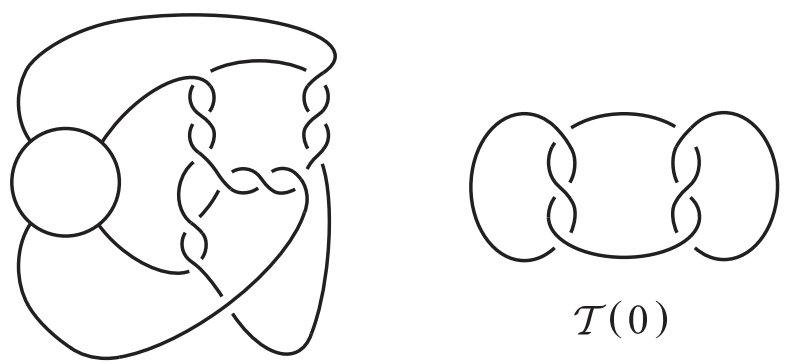

$\mathcal{T}$
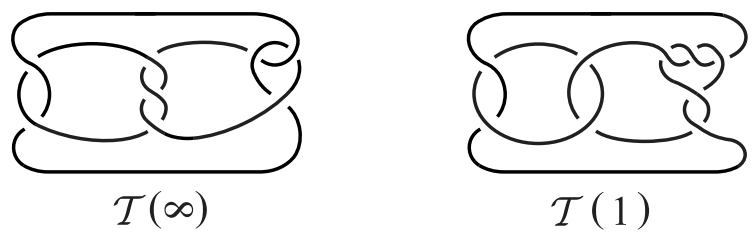

Figure 4. Tetrahedral Dehn fillings.

Lemma 4.1. The manifold $M$ admits the following Dehn fillings.

(1) $M(0)=L(3,1) \# L(3,1)$;

(2) $M(\infty)=S^{2}(2,3,3)$;

(3) $M(1)=S^{2}(2,2,7)$.

Lemma 4.2. $M$ is irreducible, $\partial$-irreducible and not Seifert fibered.

Proof. Since $M(\infty)$ and $M(1)$ are distinct prime manifolds, $M$ is irreducible.

Next, we show that $M$ is not Seifert fibered. Suppose on the contrary that $M$ is a Seifert fibered space. Since $\infty$-Dehn filling $M(\infty)$ is $S^{2}(2,3,3), M$ is $D^{2}(a, b)$ or $D^{2}(a, b, c)$ where $a$, say, must be 3 . Then $M(1)=S^{2}(2,2,7)$ is obtained by Dehn filling on $D^{2}(a, b)$ or $D^{2}(a, b, c)$. This is impossible since $a$ is 3 .

The $\partial$-irreducibility follows from the above two facts about $M$.

Theorem 4.3. The manifold $M$ is a hyperbolic manifold admitting two Dehn fillings $M\left(r_{1}\right)$ and $M\left(r_{2}\right)$ such that $M\left(r_{1}\right)$ is $L(3,1) \# L(3,1), M\left(r_{2}\right)$ is $S^{2}(2,3,3)$ and $\Delta\left(r_{1}, r_{2}\right)=1$.

Proof. By Lemmas 4.1, 4.2, we need only to show that $M$ is atoroidal.

Suppose $M$ is toroidal. Since $M(\infty)$ does not contain a non-separating torus or sphere, any essential torus in $M$ must be separating. Let $F$ be an innermost essential torus in $M$, i.e. $M=A \cup_{F} B$ where $\partial M \subseteq B$ and $A$ is atoroidal. Then $A$ and $B$ are irreducible and $\partial$-irreducible since $M$ is. By Lemma 4.1, $F$ must be compressible in $M(\infty)$ and $M(1)$. Thus $F$ is compressible in $B(\infty)$ and $B(1)$. Apply Lemma 2.5 to $B$ with slopes $\infty$ and 1. Since $M(\infty)$ and $M(1)$ don't have a lens space summand, the first case of Lemma 2.5 can't occur. Thus $B(\infty)$ and $B(1)$ are solid tori and if we let $r_{\infty}$ 

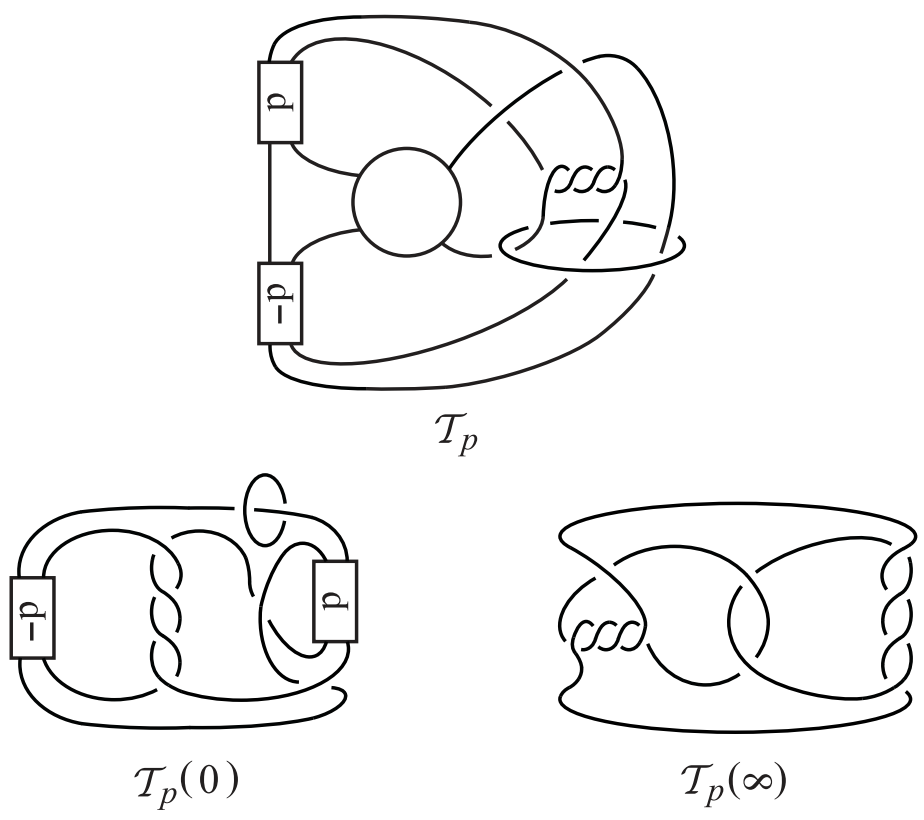

Figure 5. Octahedral Dehn fillings.

and $r_{1}$ be the slopes on $F$ corresponding to the meridians of $B(\infty)$ and $B(1)$ respectively, then $\Delta\left(r_{\infty}, r_{1}\right) \geq 4$. Also $M(\infty) \cong A\left(r_{\infty}\right)$ and $M(1) \cong A\left(r_{1}\right)$. In other words, $A\left(r_{\infty}\right)=S^{2}(2,3,3)$ and $A\left(r_{1}\right)=S^{2}(2,2,7)$.

Recall that $A$ is irreducible, $\partial$-irreducible and atoroidal. Furthermore, it is easy to see by applying an argument similar to that of the second paragraph of Lemma 4.2 that $A$ is not Seifert fibered. Therefore $A$ is hyperbolic. Since $A\left(r_{\infty}\right)=S^{2}(2,3,3)$ and $A\left(r_{1}\right)=S^{2}(2,2,7)$ with $\Delta\left(r_{\infty}, r_{1}\right) \geq 4, A$ admits two finite Dehn fillings with distance $\geq 4$. This is a contradiction to [5, Theorem 1.1].

Remark. Lemma 4.1 shows that the manifold $M$ in Theorem 4.3 is also an example of a hyperbolic manifold admitting a dihedral finite Dehn filling.

\section{OCtahedral Dehn Fillings}

In this section, we present hyperbolic manifolds admitting a reducible Dehn filling and an octahedral Dehn filling i.e. an $S^{2}(2,3,4)$-Dehn filling.

We consider the tangle $\mathcal{T}_{p}, p \geq 3$ illustrated in Figure 5. Then 0-rational tangle filling $\mathcal{T}_{p}(0)$ gives the connected sum of the Hopf link and a Montesinos link. $\infty$-rational tangle filling $\mathcal{T}_{p}(\infty)$ gives rise to a Montesinos link. Let $M_{p}$ be the double branched cover of the tangle $\mathcal{T}_{p}$. Then the following lemma follows immediately by considering the double branched covers of the above tangles or links.

Lemma 5.1. The manifolds $M_{p}$ admit the following Dehn fillings. 
(1) $M_{p}(0)=L(2,1) \# S^{2}(4, p, 2 p+1)$;

(2) $M_{p}(\infty)=S^{2}(2,3,4)$.

Theorem 5.2. The manifolds $M_{p}$ are hyperbolic manifolds admitting two Dehn fillings $M_{p}\left(r_{1}\right)$ and $M_{p}\left(r_{2}\right)$ such that $M_{p}\left(r_{1}\right)$ is $L(2,1) \# S^{2}(4, p, 2 p+1)$, $M_{p}\left(r_{2}\right)$ is $S^{2}(2,3,4)$, and $\Delta\left(r_{1}, r_{2}\right)=1$.

Proof. This follows immediately from Lemmas $5.1,5.3$ and 5.4 .

Lemma 5.3. $M_{p}$ is irreducible, $\partial$-irreducible and not Seifert fibered.

Proof. First, we show that $M_{p}$ is irreducible.

Suppose $M_{p}$ is reducible. Then it contains an essential separating sphere $S$, inducing a decomposition $M_{p}=X \# Y$ where $Y$ contains $\partial M_{p} \cdot \infty$-Dehn filling $M_{p}(\infty)$ is $X \# Y(\infty)$. By the irreducibility of $M_{p}(\infty)\left(=S^{2}(2,3,4)\right)$, $Y(\infty)$ must be $S^{3}$ and thus $X=S^{2}(2,3,4)$.

0 -Dehn filling $M_{p}(0)$ is $X \# Y(0)$. $S$ must be essential in $M_{p}(0)$. Otherwise $Y(0)$ is $S^{3}$ and $M_{p, q}(0)=X$ is reducible, a contradiction to $X=S^{2}(2,3,4)$. By the uniqueness of the prime decomposition of a 3-manifold and Lemma 5.1. $X$ must be either $L(2,1)$ or $S^{2}(4, p, 2 p+1)$. This is a contradiction because $X=S^{2}(2,3,4)$.

Suppose $M_{p}$ is a Seifert fibered space. Since $M_{p}(\infty)$ is $S^{2}(2,3,4), M_{p}$ is either $D^{2}(a, b)$ or $D^{2}(a, b, c)$ where one of $a, b, c$, say $a$, is either 2,3 , or 4 . $M_{p}$ admits a non-Seifert fibered Dehn filling $M_{p}(r)$ for at most one slope $r$, for which $M_{p}(r)$ is reducible. Furthermore such an $r$ is the slope of the Seifert fiber of $M_{p}$. Since $M_{p}(0)$ is reducible, 0 is the slope of the Seifert fiber of $M_{p}$. A fundamental group argument shows that $\pi_{1}\left(M_{p}(0)\right)$ is isomorphic to $\mathbb{Z}_{a} * \mathbb{Z}_{b}$ or $\mathbb{Z}_{a} * \mathbb{Z}_{b} * \mathbb{Z}_{c}$, where $a=2,3$ or 4 . This is a contradiction since $\pi_{1}\left(M_{p}(0)\right)=\mathbb{Z}_{2} * G$ where $G=\pi_{1}\left(S^{2}(4, p, 2 p+1)\right)$

$\partial$-irreducibility of $M_{p}$ follows from irreducibility and the fact that $M_{p}$ is non-Seifert fibered.

Lemma 5.4. $M_{p}$ is atoroidal.

Proof. Assuming the contrary, let $F$ be an essential torus in $M_{p}$. Since $M_{p}(\infty)$ does not contain a non-separating torus or sphere, $F$ must be separating. Let $M_{p}=A \cup_{F} B$ where $B$ contains $\partial M_{p}$. We may choose $F$ to be innermost, so that $A$ is atoroidal.

Since $M_{p}$ is irreducible and $\partial$-irreducible, $A$ and $B$ are also irreducible and $\partial$-irreducible. Observe by Lemma 5.1 that $M_{p}(0)$ and $M_{p}(\infty)$ are atoroidal, which implies that $F$ must be compressible in both $B(0)$ and $B(\infty)$. Then by Lemma 2.5, there are two cases to consider.

Case 1: $B$ is a cable space $C(s, t), t \geq 2$ and either 0 or $\infty$ is the cabling slope. Suppose that $\infty$ is the cabling slope of $B$. Then $B(\infty)=$ $S^{1} \times D^{2} \# L(t, s)$ and thus $M_{p}(\infty)$ has a lens space summand, which is a contradiction to Lemma 5.1. Hence the cabling slope must be 0 . Then 

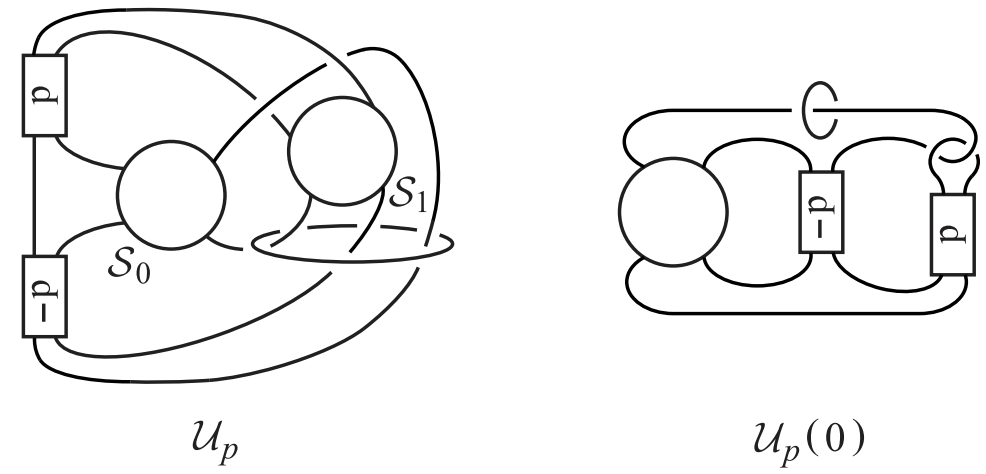

Figure 6. Rational tangle fillings on $\mathcal{U}_{p}$ obtained from $\mathcal{T}_{p}$ by removing 4-rational tangle.

$B(0)=S^{1} \times D^{2} \# L(t, s)$ and $M_{p}(0)$ has a lens space summand $L(t, s)$. Lemma 5.1 implies that $L(t, s) \cong L(2,1)$ i.e. $t=2$.

Case 2: $B(\infty)$ and $B(0)$ are $S^{1} \times D^{2}$. Let $r_{\infty}, r_{0}$ be the slopes on $F$ corresponding to the meridians of the solid tori $B(\infty), B(0)$ respectively. Then $M_{p}(\infty) \cong A\left(r_{\infty}\right)$ and $M_{p}(0) \cong A\left(r_{0}\right)$. Correspondingly, $A\left(r_{\infty}\right)=$ $S^{2}(2,3,4)$ and $A\left(r_{0}\right)$ is reducible. Also $\Delta\left(r_{\infty}, r_{0}\right) \geq 4$ by Lemma 2.5. Recall that $A$ is an irreducible, $\partial$-irreducible and atoroidal 3 -manifold with torus boundary $F$. However, the non-Seifert fiberedness of $A$ follows from an argument similar to that proving that $M_{p}$ is not a Seifert fibered space in Lemma 5.3. This implies that $A$ is hyperbolic, and admits a reducible Dehn filling and a finite Dehn filling with distance greater than 1 . This is a contradiction to [2, Theorem 1].

We have shown from Cases 1,2 that $B$ is the cable space $C(s, 2)$ with cabling slope 0 . Hence $M_{p}=A \cup_{F} C(s, 2)$.

Now we consider the tangle $\mathcal{U}_{p}$ with two tangle spheres $\mathcal{S}_{0}, \mathcal{S}_{1}$ shown in Figure 6. Let $N_{p}, \partial_{0} N_{p}, \partial_{1} N_{p}$ be the double branched covers of $\mathcal{U}_{p}, \mathcal{S}_{0}, \mathcal{S}_{1}$ respectively. Then observe that the tangle $\mathcal{T}_{p}$ in Figure 5 is obtained from $\mathcal{U}_{p}$ by 4 -rational tangle filling on $\mathcal{S}_{1}$. As double branched covers, $M_{p}$ is obtained from $N_{p}$ by 4 -Dehn filling on $\partial_{1} N_{p}$ i.e. $M_{p}=N_{p}(4)$. Hence $N_{p}(4)=$ $A \cup_{F} C(s, 2)$, which is toroidal.

Claim. F separates the two boundaries $\partial_{0} N_{p}$ and $\partial_{1} N_{p}$ of $N_{p}$. Therefore $A$ has the boundary $\partial_{1} N_{p}$.

Proof. Since $B$ is the cable space $C(s, 2)$ with cabling slope $0, B(0)=$ $S^{1} \times D^{2} \# L(2, s)$. Let $V$ be the solid torus summand of $B(0)$ and $J$ the core of $V$. Note that $\partial V=F$ since $\partial B(0)=F$. Consider $M_{p}(0)=$ $L(2,1) \# S^{2}(4, p, 2 p+1)$. Since $M_{p}(0)=A \cup_{F} B(0)$ and $B(0)=V \# L(2, s)$, it follows that $S^{2}(4, p, 2 p+1)$ is obtained from $A$ by attaching $V$ along $F$ i.e. $S^{2}(4, p, 2 p+1)=A \cup_{F} V$. Therefore $S^{2}(4, p, 2 p+1)$ contains $V$.

Recall that the tangle $\mathcal{U}_{p}$ is obtained from the tangle $\mathcal{T}_{p}$ by removing the 4-rational tangle. Let $R$ be the 4-rational tangle. Let $W$ be the solid 
torus in $M_{p}$ which is the double branched cover of $R$, and $K$ the core of $W$. Note that $\partial R=\mathcal{S}_{1}$. Therefore $\partial W=\partial_{1} N_{p}$. Recall from Figure 5 that $\mathcal{T}_{p}(0)$ is the connected sum of the Hopf link and a Montesinos link. Then $S^{2}(4, p, 2 p+1)$ summand in $M_{p}(0)$ is the double branched cover of the Montesinos link in $\mathcal{T}_{p}(0)$. Furthermore we can observe from Figure 5 that -1/4-rational tangle in the Montesinos link comes directly from the 4-rational tangle $R$. Therefore $S^{2}(4, p, 2 p+1)$ contains $W$ since $W$ is the double branched cover of $R$.

We have seen that $S^{2}(4, p, 2 p+1)$ contains $V$ and $W$ with $\partial V=F$ and $\partial W=\partial_{1} N_{p}$. Perturbing the cores $J$ and $K$ of $V$ and $W$, we may assume that $K$ doesn't intersect $J$ in $S^{2}(4, p, 2 p+1)$. In other words, $W$ doesn't intersect $V$. Thus $\partial_{1} N_{p}$ doesn't intersect $V$. Since $S^{2}(4, p, 2 p+1)=A \cup_{F} V$, $\partial_{1} N_{p}$ is contained in $A$, as desired.

We perform a 0 -rational tangle filling on $\mathcal{U}_{p}$ along $\mathcal{S}_{1}$, which gives the connected sum of the Hopf link and some Montesinos tangle as described in Figure6. Then the corresponding double branched cover $N_{p}(0)$ is $L(2,1) \# D^{2}(p$, $2 p+1)$. We claim that $N_{p}$ is hyperbolic. As long as the claim holds, the hyperbolic manifold $N_{p}$ admits the reducible Dehn filling $N_{p}(0)$ and the toroidal Dehn filling $N_{p}(4)$, and $\Delta(0,4)=4$. This is a contradiction to [15, Theorem 1.1] or [17, Theorem 1].

Claim. $N_{p}$ is hyperbolic.

Proof. $N_{p}$ admits two exceptional Dehn fillings $N_{p}(0)=L(2,1) \# D^{2}(p, 2 p+$ 1) and $N_{p}(4)=A \cup_{F} C(s, 2)$, which is toroidal. Note that by Lemma 5.3 $N_{p}(4)$ is irreducible, $\partial$-irreducible and non-Seifert fibered.

Suppose that $N_{p}$ is not irreducible. In other words, there is an essential sphere $S$ in $N_{p}$. $S$ must be separating since $N_{p}(4)$ does not contain a nonseparating sphere, so it induces a decomposition $N_{p}=X \# Y$ with $\partial_{1} N_{p} \subseteq Y$. Then $N_{p}(0)=X \# Y(0)$ and $N_{p}(4)=X \# Y(4)$. Since $N_{p}(4)$ is irreducible, $Y(4)$ is $S^{3}$ and thus $N_{p}(4) \cong X$. Hence $X$ is irreducible and toroidal. On the other hand, if $S$ is inessential in $N_{p}(0)$, then $Y(0)$ must be $S^{3}$, which implies that $X\left(\cong N_{p}(0)\right)$ is reducible, a contradiction. If $S$ is essential in $N_{p}(0)$, then $X$ must be either $L(2,1)$ or $D^{2}(p, 2 p+1)$, neither of which is toroidal, a contradiction.

$N_{p}$ is not Seifert fibered since it has two non-Seifert fibered Dehn fillings $N_{p}(0)$ and $N_{p}(4)$. By irreducibility and the fact that it is non-Seifert fibered, $N_{p}$ is $\partial$-irreducible. To complete the proof, we need only to show that $N_{p}$ is atoroidal.

Suppose $N_{p}$ contains an essential torus $G$. Then $G$ must be separating since $N_{p}(0)$ does not contain a non-separating torus or sphere. Let $N_{p}=$ $D \cup_{G} E$ where the filling torus $\partial_{1} N_{p} \subseteq E$ and $G$ is chosen to be innermost with respect to $\partial_{1} N_{p}$, so that $D$ is atoroidal. Then $D$ and $E$ are irreducible and $\partial$-irreducible since $N_{p}$ is. $G$ is compressible in $N_{p}(0)$ and thus in $E(0)$ 
since $N_{p}(0)$ is atoroidal. Hence $E(0)=S^{1} \times D^{2} \# W$ for some 3 -manifold $W$. Let $s_{0}$ be the slope on $G$ corresponding to the meridian of the solid torus summand of $E(0)$. Then $N_{p}(0)=D\left(s_{0}\right) \# W$.

Suppose $G$ is compressible in $N_{p}(4)$. By Lemma $2.4 E$ is a cable space $C(u, v)$ with $v \geq 2$ with cabling slope $\infty$. Since $\Delta(\infty, 0)=\Delta(\infty, 4)=1$, $E(0)$ and $E(4)$ are solid tori $S^{1} \times D^{2}$. Let $s_{4}$ be the slope on $G$ corresponding to the meridian of $E(4)$. Then $N_{p}(0)=D\left(s_{0}\right)$ and $N_{p}(4)=D\left(s_{4}\right)$, which are reducible and toroidal respectively. Moreover by 11, Lemma 3.1], $\Delta\left(s_{0}, s_{4}\right)=v^{2} \Delta(0,4)$ and thus $\Delta\left(s_{0}, s_{4}\right) \geq 16$. Recall that $D$ is irreducible, $\partial$-irreducible and atoroidal. However $D$ is also not Seifert fibered. Otherwise, $D$ admits only one non-Seifert fibered Dehn filling, which is a contradiction since $D\left(s_{0}\right)$ and $D\left(s_{4}\right)$ are not Seifert fibered. Hence we have obtained a hyperbolic manifold $D$ which admits a reducible Dehn filling $D\left(s_{0}\right)$ and a toroidal Dehn filling $D\left(s_{4}\right)$ with distance $\geq 16$. This is a contradiction to [15, Theorem 1.1] or [17, Theorem 1].

Suppose $G$ is incompressible in $N_{p}(4)$. Since $N_{p}(4)$ is not a Seifert fibered space, there is only one essential torus in $N_{p}(4)=A \cup_{F} C(s, 2)$ up to isotopy. Thus $G$ is isotopic to $F$. Since $N_{p}(4)=D \cup_{G} E(4), E(4)$ is homeomorphic to either $A$ or $C(s, 2)$. However Claim 5 says that $A$ contains $\partial_{1} N_{p}$. Since $E(4)$ also contains $\partial_{1} N_{p}, E(4)$ is homeomorphic to $A$. Therefore $D$ is homeomorphic to $C(s, 2)$.

Recall that $N_{p}(0)=D\left(s_{0}\right) \# W$. Since $D=C(s, 2), D\left(s_{0}\right)$ is either $D^{2}(2, a)$ or $S^{1} \times D^{2} \# L(2, s)$ for some positive integer $a$. Thus $N_{p}(0)=$ $D^{2}(2, a) \# W$ or $S^{1} \times D^{2} \# L(2, s) \# W$. However either case is impossible since $N_{p}(0)=L(2,1) \# D^{2}(p, 2 p+1)$ where $p \geq 3$. This completes the proof of the claim.

The claim completes the proof of the lemma.

\section{ICOSAHEDRAL Dehn FILLINGS}

In this section, we present hyperbolic manifolds which admit a reducible Dehn filling and a Dehn filling of type $S^{2}(2,3,5)$ at distance 1 .

Consider the tangles $\mathcal{T}_{p, q}$ shown in Figure 7 , where $p, q$ are integers satisfying $p \neq 0, \pm 1, q \neq 0,(p, q) \neq( \pm 2, \pm 1)$. The tangles $\mathcal{T}_{p, q}$ were constructed by Lee [14, Section 4]. He used the tangles $\mathcal{T}_{p, q}$ to show that there are hyperbolic 3-manifolds admitting an $S^{1} \times S^{2}$ Dehn filling and a toroidal Dehn filling with distance 2. (More explicitly, the double branched covers of $\mathcal{T}_{p, q}(-1 / 2)$ and $\mathcal{T}_{p, q}(\infty)$ are $S^{1} \times S^{2}$ and a toroidal 3-manifold respectively.) Let $M_{p, q}$ be the double branched cover of $\mathcal{T}_{p, q}$. Then $M_{p, q}$ with $p, q$ as above is hyperbolic by [14].

We consider several rational tangle fillings on $\mathcal{T}_{p, q}, \mathcal{T}_{p, q}(-1 / 2), \mathcal{T}_{p, q}(0)$, $\mathcal{T}_{p, q}(-1)$, which are depicted in Figure 7 . Then the following lemma follows from Figure 7, considering double branched covers.

Lemma 6.1. The manifold $M_{p, q}$ admits the following Dehn fillings. 

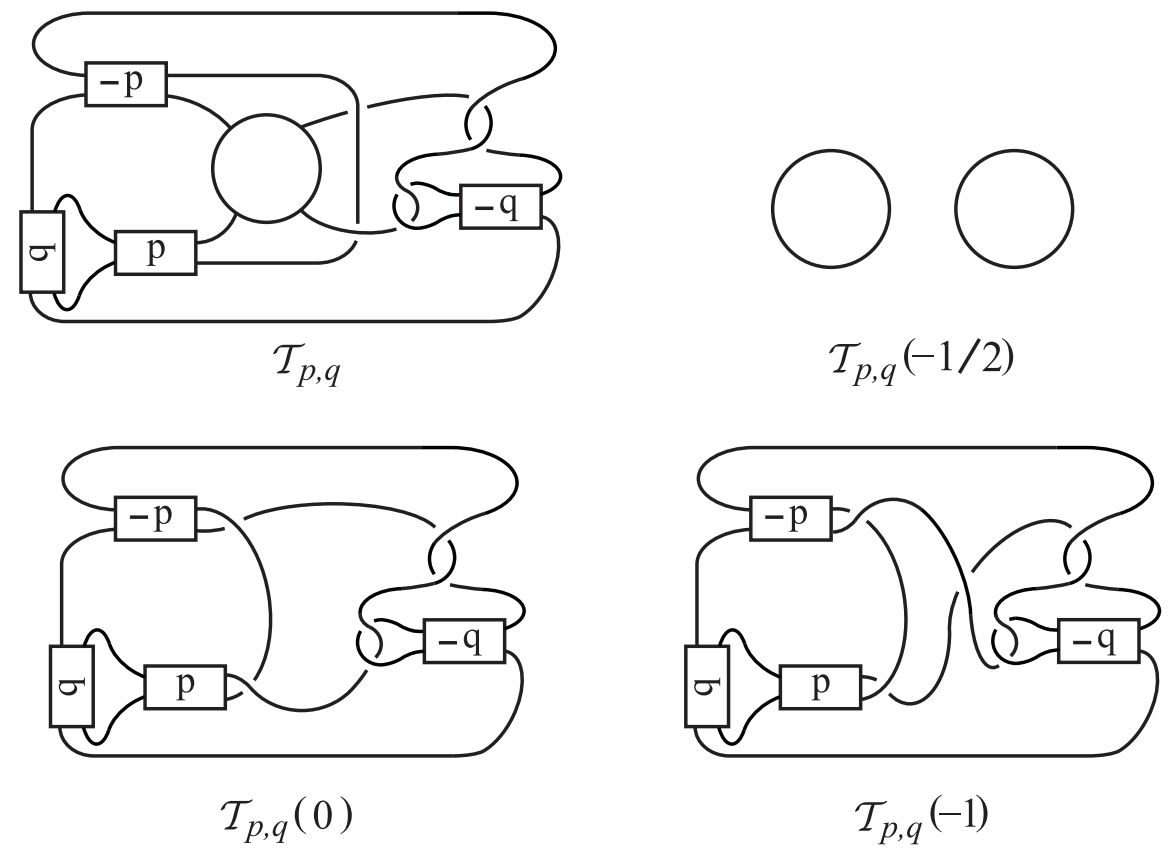

Figure 7. Icosahedral Dehn fillings.

(1) $M_{p, q}(-1 / 2)=S^{1} \times S^{2}$;

(2) $M_{p, q}(0)=S^{2}(|p-1|,|2 q-1|,|p q+q-1|)$;

(3) $M_{p, q}(-1)=S^{2}(|p+1|,|2 q+1|,|p q-q-1|)$.

Theorem 6.2. The manifolds $M_{p, q},(p, q)=( \pm 3, \mp 1),( \pm 4, \pm 1)$, are hyperbolic manifolds admitting two Dehn fillings $M_{p, q}\left(r_{1}\right)$ and $M_{p, q}\left(r_{2}\right)$, such that $M_{p, q}\left(r_{1}\right)$ is $S^{1} \times S^{2}$ i.e. reducible, $M_{p, q}\left(r_{2}\right)=S^{2}(2,3,5)$ and $\Delta\left(r_{1}, r_{2}\right)=1$.

Proof. As mentioned above, the manifolds $M_{p, q}$ are hyperbolic by [14].

If $(p, q)=(3,-1),(-4,-1)$, then $M_{p, q}(0)=S^{2}(2,3,5)$. If $(p, q)=$ $(-3,1),(4,1)$, then $M_{p, q}(-1)=S^{2}(2,3,5)$. Also $\Delta(0,-1 / 2)=1$ and $\Delta(-1$, $-1 / 2)=1$. We are done.

We present another hyperbolic manifold admitting an icosahedral Dehn filling. Consider the tangle $\mathcal{T}$ and the three rational tangle fillings $\mathcal{T}(0)$, $\mathcal{T}(\infty), \mathcal{T}(1)$ shown in Figure $8, \mathcal{T}(0)$ is the connected sum of the trefoil knot and the $(2,4)$ torus link, while $\mathcal{T}(\infty)$ and $\mathcal{T}(1)$ are Montesinos links. Let $M$ be the double branched cover of $\mathcal{T}$. Considering the double branched covers of these, we have the following lemma.

Lemma 6.3. The manifold $M$ admits three Dehn fillings as follows;

(1) $M(0)=L(3,1) \# L(4,1)$;

(2) $M(\infty)=S^{2}(2,3,5)$;

(3) $M(1)=S^{2}(2,3,7)$. 

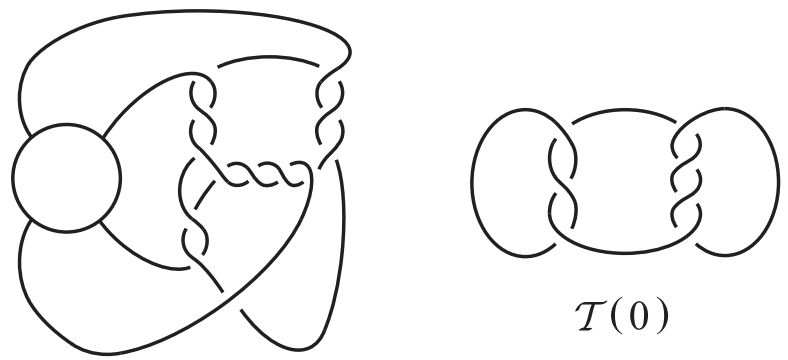

$\mathcal{T}$
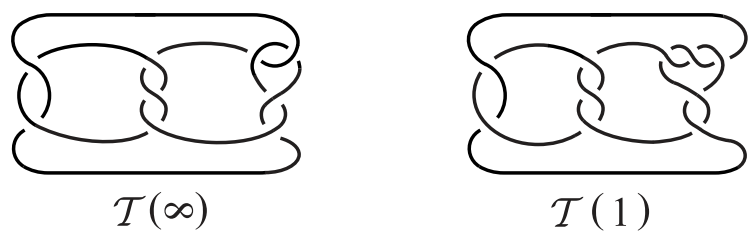

Figure 8. Another icosahedral Dehn fillings.

Theorem 6.4. The manifold $M$ is a hyperbolic manifold. Thus $M$ is another example of a hyperbolic manifold having a reducible and an icosahedral Dehn filling.

Proof. Apply the same argument of Lemma 4.2 for irreducibility and $\partial$ irreducibility of $M$, replacing $S^{2}(2,3,3), S^{2}(2,2,7)$ by $S^{2}(2,3,5), S^{2}(2,3,7)$ respectively.

Assume that $M$ is Seifert fibered. Then $M$ must be homeomorphic to $D^{2}(2,3)$ by Lemma $6.3(2),(3)$. Observe that $M(r)$ is Seifert fibered for all but at most one slope $r$, for which $M(r)$ is reducible. When $M(r)$ is reducible, $r$ is the slope of the Seifert fiber of $M$. Hence Lemma 6.3 implies that $M(0)=L(3,1) \# L(4,1)$ is obtained by Dehn filling on $M=D^{2}(2,3)$ with the slope 0 corresponding to the Seifert fiber of $M$. This is impossible by a fundamental group argument.

In order to prove that $M$ is hyperbolic, it remains to show that $M$ is atoroidal. Suppose $M$ is toroidal. Since $M(\infty)$ does not contain a nonseparating torus or sphere, any essential torus in $M$ must be separating. Let $F$ be an innermost essential torus in $M$, i.e. $M=A \cup_{F} B$ where $\partial M \subseteq B$ and $A$ is atoroidal. Then $A, B$ are irreducible and $\partial$-irreducible. By Lemma 6.3. $F$ must be compressible in $M(0), M(\infty)$ and $M(1)$ and thus in $B(0), B(\infty)$ and $B(1)$. Applying Lemma 2.5 to $B$ with the two slopes $0, \infty$, we have two cases to consider.

Case 1: $B$ is a cable space $C(s, t), t \geq 2$ with cabling slope 0 or $\infty$. Since $M(\infty)=S^{2}(2,3,5)$, which does not have a lens space summand, $\infty$ cannot be the cabling slope of $B$. Therefore 0 is the cabling slope. Then $B(0)=S^{1} \times D^{2} \# L(t, s)$ and $B(\infty)=S^{1} \times D^{2}$. Also $B(1)=S^{1} \times D^{2}$ since $\Delta(0,1)=1$. Note that $M(0)=A \cup_{F} B(0), M(\infty)=A \cup_{F} B(\infty)$ and 
$M(1)=A \cup_{F} B(1)$. If we let $r_{0}, r_{\infty}$ and $r_{1}$ be the slopes on $F$ corresponding to the meridians of the solid tori of $B(0), B(\infty)$ and $B(1)$ respectively, then $M(0)=A\left(r_{0}\right) \# L(t, s), M(\infty)=A\left(r_{\infty}\right)$ and $M(1)=A\left(r_{1}\right)$. Lemma 6.3 implies that $A\left(r_{0}\right) \cong L(3,1)$ or $L(4,1), A\left(r_{\infty}\right) \cong S^{2}(2,3,5), A\left(r_{1}\right) \cong$ $S^{2}(2,3,7)$ and $t=3$ or 4 . Furthermore, from the proof of Lemma 2.5, we know that $\Delta\left(r_{0}, r_{\infty}\right) \geq t$. Thus $\Delta\left(r_{0}, r_{\infty}\right) \geq 3$.

We have shown that the irreducible, $\partial$-irreducible and atoroidal manifold $A$ admits two Dehn fillings $A\left(r_{0}\right) \cong L(3,1)$ or $L(4,1), A\left(r_{\infty}\right) \cong S^{2}(2,3,5)$ and $\Delta\left(r_{0}, r_{\infty}\right) \geq 3$. This is a contradiction to [ 3 , Theorem 1.1], provided that $A$ is not Seifert fibered (thus $A$ is hyperbolic).

To complete Case 1, we need to show that $A$ is not Seifert fibered. Suppose $A$ is Seifert fibered. Then since $A\left(r_{\infty}\right) \cong S^{2}(2,3,5)$ and $A\left(r_{1}\right) \cong S^{2}(2,3,7)$, $A$ must be homeomorphic to $D^{2}(2,3)$, which is homeomorphic to the complement of the trefoil knot in $S^{3}$ i.e. the $(2,3)$-torus knot. Also $A\left(\cong D^{2}(2,3)\right)$ admits the lens space Dehn filling $A\left(r_{0}\right)(=L(3,1)$ or $L(4,1))$. However this is impossible by [9, Lemma 7.4].

Case 2: $B(0)$ and $B(\infty)$ are $S^{1} \times D^{2}$. Note that by applying Lemma 2.5 to two slopes 0 and 1 , and by knowing the fact that $M(1)$ doesn't have a lens space summand, we can assume $B(1)$ to be $S^{1} \times D^{2}$. Let $r_{0}, r_{\infty}$ and $r_{1}$ be the slopes on $F$ corresponding to the meridians of $B(0), B(\infty)$ and $B(1)$ respectively. Then $M(0)=A\left(r_{0}\right)(=L(3,1) \# L(4,1)), M(\infty)=A\left(r_{\infty}\right)$ $\left(=S^{2}(2,3,5)\right)$ and $M(1)=A\left(r_{1}\right)\left(=S^{2}(2,3,7)\right)$. Also Lemma 2.5 shows that $\Delta\left(r_{0}, r_{\infty}\right) \geq 4$. Then it is easy to see by applying the same argument of the second paragraph of this theorem that $A$ is not Seifert fibered and thus hyperbolic. Then considering the manifold $A$, we get a contradiction to [2, Theorem 1]. This completes the proof.

\section{REFERENCES}

[1] S. BOYER, M. CULLER, P.B. SHALEN and X. ZHANG. Characteristic subsurfaces, character varieties and Dehn fillings. Preprint, 2006. arXiv:math.GT/0611669.

[2] S. BOYER, C. MCA. GORDON and X. ZHANG. Reducible and finite Dehn fillings. Preprint, 2007. arXiv:0710.3786.

[3] S. BOYER and X. ZHANG. Finite Dehn surgery on knots. J. Amer. Math. Soc. 9 (1996), 1005-1051

[4] S. BOYER and X. ZHANG. On Culler-Shalen seminorms and Dehn fillings. Ann. of Math. 148 (1998), 737-801.

[5] S. BOYER and X. ZHANG. A proof of the finite filling conjecture. J. Diff. Geom. 59 (2001), 87-176.

[6] M. CUllER, C. MCA. GORDON, J. LUECKE, and P.B. SHALEN. Dehn surgery on knots. Ann. of Math. 125 (1987), 237-300.

[7] M. EUDAVE-MUÑOZ and Y.Q. WU. Nonhyperbolic Dehn fillings on hyperbolic 3-manifolds. Pacific J.Math. 190 (1999), 261-275.

[8] D. GABAI. Surgery on knots in solid tori. Topology, (28) (1989), 261-275.

[9] C. MCA. GORDON. Dehn surgery and satellite knots. Trans. Amer. Math. Soc. 275 (1983), 687-708. 
[10] C. MCA. GORDON. Small surfaces and Dehn filling. Geometry and Topology Monographs Vol. 2, Proceedings of the Kirbyfest (1999), 177-199.

[11] C. MCA. GORDON and R.A. LITHERLAND. Incompressible planar surfaces in 3-manifolds. Topology Appl. 18 (1984), 121-144.

[12] C. MCA. GORDON and J. LUECKE. Only integral Dehn surgeries can yield reducible manifolds. Math. Proc. Camb. Phil. Soc. 102 (1987), 94-101.

[13] C. MCA. GORDON and Y.Q. WU. Toroidal Dehn fillings on hyperbolic 3-manifolds. Preprint, 2005. to appear in Memoirs Amer. Math. Soc. arXiv:math.GT/0512038

[14] S. LEE. Toroidal Dehn surgeries on Knots in $S^{1} \times S^{2}$. J. Knot Theory Ramifications 14 (2005), 657-664.

[15] S. OH. Reducible and toroidal manifolds obtained by Dehn fillings. Topology Appl. 75 (1997), 93-104.

[16] M. SCHARLEMANN. Producing reducible 3-manifolds by surgery on a knot. Topology, 29 (1990), 481-500.

[17] Y.Q. WU. Dehn fillings producing reducible manifolds and toroidal manifolds. Topology 37 (1998), 95-108.

Department of Mathematics, University of Texas at Austin, 1 University Station C1200 Austin, TX 78712-0257

E-mail address: skang@math.utexas.edu 